\title{
Implantation of Durable VAD during COVID-19: An Epicenter Experience
}

\author{
Scott Stewart, DNP*, David Silber, DO, Bernard Kim, MD, Kumar Satya, MD, George Batsides, MD, \\ Kanika Mody, MD, and Mark Anderson, MD
}

Hackensack University Medical Center, Hackensack, New Jersey, USA

*Corresponding author: Scott Stewart, DNP, APNC, CNL, Hackensack University Medical Center, 20

Prospect Ave, Suite 201, Hackensack, New Jersey 07601, USA, Tel: 201-741-4719

\begin{abstract}
Ventricular Assist Devices (VAD) serves as an advanced treatment modality for end stage systolic heart failure patients. Implantation of VAD requires a multidisciplinary team approach with in depth family meetings and education sessions to ensure a successful outcome. During the COVID-19 pandemic, multiple new barriers were identified to allow for traditional VAD implantation and post-operative care. Modifications to current processes needed to be developed to accommodate a safe and comprehensive plan. We describe a two patient experience of implantation of durable VAD from the initial United States epicenter area who underwent successful device therapy.
\end{abstract}

\section{Keywords}

Ventricular assist device, COVID-19

\section{Introduction}

Ventricular Assist Devices (VAD) serves as an advanced treatment modality for end stage systolic heart failure patients [1]. Device implantation requires a comprehensive team approach that includes an in-depth workup and evaluation process [2]. Often this includes multiple family meetings and in-person training preoperatively. In March 2020, the northeast tristate area of the United States experienced an extreme stress on the healthcare system as the initial epicenter of the North American COVID-19 outbreak [3]. During this difficult time, our heart failure program managed two end-stage cardiomyopathy patientswho were fortunately $\mathrm{CO}$ VID-19 negative but had progressive heart failure which required evaluation and successful destination therapy (DT) LVAD implantation thru a unique modified approach.

\section{Patient Care Experience}

Patient $A$ is a 60 -year-old male with a past medical history of nonischemic dilated cardiomyopathy who was referred to the heart failure clinic for advanced therapies from his primary cardiologist. He was admitted on March 5, 2020 just prior to the outbreak of COVID-19 due to failure of guideline-directed medical therapy and for hemodynamic evaluation. He was started on escalating doses of inotropic support and a right heart catheterization was performed revealing hemodynamics confirming low output heart failure and impending cardiogenic shock. He underwent placement of an Impella 5.5 pump (Abiomed, Danvers, MA) via axillary approach for acute left ventricular support on $3 / 27 / 20$. During this time, COVID-19 began to flourish in this area and restrictions were initiated including no visitors to the facility, redeployment of nurses and physicians including the core VAD team as well as clustering of patient acuity and COVID status. While workup began for device therapy, COVID-19 cases continued to surge in our facility taking all available ICU capacity requiring non-COVID ICU and surgical stepdown level of care to be relocated to the post-anesthesia recovery unit (PACU). Patient was evaluated for device therapy but deemed high risk for acute right heart failure based on hemodynamic parameters and echocardiogram images. While ideal for this patient, transplantation was not an option as local centers paused this procedure for fears of infectious concerns and lack of resources. The decision was made to proceed with implantation of a HeartMate III DT-LVAD (Abbott, Chicago, IL). Post-operatively he was challenged with acute right ventricular

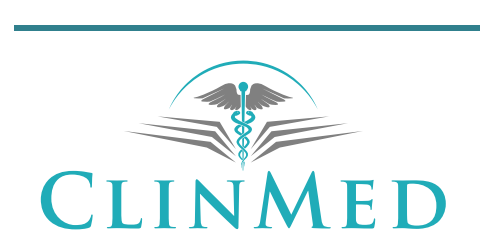

INTERNATIONAL LIBRARY
Citation: Stewart S, Silber D, Kim B, Satya K, Batsides G, et al. (2020) Implantation of Durable VAD during COVID-19: An Epicenter Experience. Int J Clin Cardiol 7:206. doi.org/10.23937/2378-2951/1410206 Accepted: December 10, 2020: Published: December 12, 2020

Copyright: (c) 2020 Stewart S, et al. This is an open-access article distributed under the terms of the Creative Commons Attribution License, which permits unrestricted use, distribution, and reproduction in any medium, provided the original author and source are credited. 
failure requiring a prolonged inotropic support course as well as acute delirium as he could not leave a windowless PACU and family visitation was completely suspended. Due to continued surging of COVID cases, he could not be discharged to a VAD trained rehabilitation site and was sent home after a very prolonged post-operative stay.

Patient B is a 79-year-old male transferred for advanced therapy evaluation in May after presenting with acute decompensated heart failure from prolonged nonischemic cardiomyopathy. He had prior admissions for presumed pneumonia in March 2020 and was COVID negative at that time. He was discharged to home on inotropic support with plans for VAD evaluation outpatient when COVID risk was reduced. Unfortunately he was readmitted shortly after discharge for heart failure exacerbation requiring urgent VAD evaluation. Upon his readmission, he was required to be in a COVID-19 unit under strict isolation until multiple negative PCR tests resulted. Although transplantation had resumed by this point, his age precluded this procedure. He was evaluated for device therapy and a Heart Mate III was implanted at the end of May. At this time, post-operative care had resumed in the traditional ICU setting however restrictions still existed for visitation. Several of the core VAD team staff remained redeployed creating the need for virtual multidisciplinary team meetings and training sessions.

\section{Discussion}

Traditionally implantation of a DT-VAD requires extensive workup, in-person family meetings, multidisciplinary appointments, and elective admission, however due to restrictions from the pandemic alternative modalities were required to achieve similar standards [1]. Due to visitation restrictions, all pre-VAD evaluation for families and caregivers was performed via video conference applications. This allowed for multiple providers to meet with families in a structured timely fashion as well as adhere to appropriate quarantine guidelines. Our palliative care team was able to join from their respective areas and all information was displayed on projections from computer screens as opposed to physical in-person meetings. For one of our patients, families resided in states with travel restrictions and therefore despite hospital permissions, they could not be present. This utilization of technology allowed for family participation in clinical care but from a distance. The multidisciplinary team, which typically draw representatives from every department were reduced to only cardiology, surgery, palliative and care and social work meeting at the patient's bedside to adhere to social distancing. Additionally, our core VAD team was redeployed to care for COVID patients requiring alterations to the typical practice patterns and limited our ancillary support and staff resources. To meet the high demands of COVID-19 intensive care needs, our facility needed to modify physical space including the need for transitioning the cafe- teria to a COVID-19 unit and ceasing of all elective cases [4]. Our cardiothoracic surgery ICU and stepdown floors were also transitioned to COVID-19 units thus creating the struggle for these non-COVID in-patient procedures. The PACU was transitioned to a non-COVID ICU and stepdown unit for surgical patients to accommodate longer term recovery that would have traditionally been done on a stepdown floor. Delirium was a challenge given the unit was without windows and required creation of sleep/wake cycles with combination of lighting and medication adjustments. Caregiver device therapy education is typically performed over multiple sessions including hands on teaching for sterile driveline care. To simulate this experience, caregivers were trained using video support first for home review. On subsequent encounters they met in the out-patient clinic using mock equipment with a mannequin that could be disinfected. Families were granted case by case visitation which allowed for one person to visit only once for an inperson education session with the patient and their actual equipment. Post VAD visits were arranged via video conferencing apps to allow for telehealth visits for successful patient management.

\section{Conclusion}

While this was only a two patient experience, new technological advances and creativity from our team allowed for successful implantation of urgent DT-VAD during the COVID-19 pandemic. Lessons learned during this time can be applicable for future challenges that may prevent traditional VAD workup and management. This experience demonstrated the resiliency of a VAD team to provide the highest level of care for an extremely sick but recoverable patient population.

\section{Authors Declaration}

There was no funding for this article. Dr. Mark Anderson and Dr. George Batsides are both speakers and current members of the advisory board for Abiomed which is mentioned in this article however do not believe this to contribute to bias in this article. The remaining authors do not have any disclosures.

\section{References}

1. Yancy CW, Jessup M, Bozkurt B, Butler J, CaseyJr DE, et al. (2017) 2017 ACC/AHA/HFSA Focused Update of the 2013 ACCF/AHA Guideline for the Management of Heart Failure: A Report of the American College of Cardiology/ American Heart Association Task Force on Clinical Practice Guidelines and the Heart Failure Society of America. Circulation 136: e137-e161.

2. Guglin M, Zucker MJ, Borlaug BA, Breen E, Cleveland J, et al. (2020) Evaluation for heart transplantation and LVAD implantation: JACC Council Perspectives. J Am Coll Cardiol 75: 1471-1487.

3. New Jersey Hospital Association (2020) Report: COVID-19 Results in Unprecedented Fiscal Challenges for NJ Hospitals.

4. Vecchione A (2020) Hackensack University Medical Center converts cafeteria to increase capacity. NJBiz. 